\title{
Geometrical symmetries of the Universal equation
}

\author{
V. DERJAGIN and A. LEZNOV \\ Institute for High Energy Physics, 142284 Protvino, \\ Moscow Region, Russia \\ Submitted by W. FUSHCHYCH \\ Received September 10, 1994
}

\begin{abstract}
It is shown that the group of geometrical symmetries of the Universal equation of $D$-dimensional space coincides with $S L(D+1, R)$.
\end{abstract}

\section{Introduction}

The Universal equation was first introduced in a series of papers by D.B.Fairlie and collaborators [1] with the goal of constructing the example of an integrable system in the space of arbitrary dimensions. In the simplest case $(D=2)$ this equation is exactly the Batemann equation [2], which in its turn is closely connected with the equation of Monge [2]. Later it was understood that this equation may be related to the problem of construction of local solutions of the bi-harmonical equation. In particular, for the case $D=3$ it is connected with the bi-harmonical equation in usual four-dimensional space [3]. This problem, in turn, is in closed connection with the problem of constructing the instanton solution in four-dimensional Yang-Mills theory [4].

In this note we want to show that the Universal equation is invariant with respect to fractional-linear transformations in $D+1$ dimensions, and consequently that the group of geometrical symmetries of this equation coincides with $S L(D+1, R)$.

\section{General properties of the Universal equation}

The original form of the Universal equation in the space of $D$ dimensions is the following

$$
\operatorname{Det}_{D+1}\left(\begin{array}{cc}
0 & \phi_{i} \\
\phi_{j} & \phi_{i, j}
\end{array}\right)=0 \quad(D \geq i, j \geq 1)
$$


where $\phi_{i} \equiv \frac{\partial \phi}{\partial x_{i}}, \phi_{i, j} \equiv \frac{\partial^{2} \phi}{\partial x_{i} \partial x_{j}}, \phi$ is an unknown function, and $x_{i}$-the coordinates of the
space.

This equation possesses remarkable properties. There is an infinite number of Lagrange functions from which (2.1) arises by the known rules. There is an infinite number of conservations laws for this equation. It is possible to find the general solution of (2.1) with the help of the Legendre transformation [1].

We shall call all transformations of coordinates $x_{i}^{\prime}=f_{i}\left(x_{1}, x_{2}, \ldots x_{D}\right)$, with respect to which the equation (2.1) conserves its form, geometrical transformations.

\section{The condition of invariance}

Let us suppose that $x_{i}^{\prime}$ are new coordinates. The derivatives with respect to old coordinates which are interesting for us are connected with the new ones by the usual formulae

$$
\phi_{i}=\sum_{s} \frac{\partial x_{s}^{\prime}}{\partial x_{i}} \phi_{s}^{\prime} \quad \phi_{i j}=\sum_{s k} \frac{\partial x_{s}^{\prime}}{\partial x_{i}} \frac{\partial x_{k}^{\prime}}{\partial x_{j}} \phi_{s k}+\sum_{s} \frac{\partial^{2} x_{s}^{\prime}}{\partial x_{i} \partial x_{j}} \phi_{s}^{\prime} .
$$

The condition of invariance of (2.1) is equivalent to a system

$$
\frac{\partial^{2} x_{s}^{\prime}}{\partial x_{i} \partial x_{j}}=B_{i} \frac{\partial x_{s}^{\prime}}{\partial x_{j}}+B_{j} \frac{\partial x_{s}^{\prime}}{\partial x_{i}}
$$

where $B_{i}$ are some functions which must be defined from the solution of (3.2) together with $x_{s}^{\prime}$.

In the case $i=j$ it follows immediately from (3.2)

$$
B_{i}=\frac{1}{2} \frac{\partial}{\partial x_{i}} \ln \left(\frac{\partial x_{s}^{\prime}}{\partial x_{i}}\right)
$$

for all $s$, or taking the first integral we obtain

$$
\frac{\partial x_{1}^{\prime}}{\partial x_{i}}=f_{s}^{(i} \frac{\partial x_{s}^{\prime}}{\partial x_{i}}
$$

where the symbol $f^{(i}$ means that the function $f$ is independent of an argument $x_{i}$. Integrating (3.3) once more we obtain as a result

$$
x_{1}^{\prime}=f_{s}^{(i} x_{s}^{\prime}+F_{s}^{(i}, \quad s=2, . ., D .
$$

Substituting $B_{i}$ into (3.2) we obtain

$$
2 \frac{\partial x_{s}^{\prime}}{\partial x_{i}} \frac{\partial x_{s}^{\prime}}{\partial x_{j}} \frac{\partial^{2} x_{s}^{\prime}}{\partial x_{i} \partial x_{j}}=\left(\frac{\partial x_{s}^{\prime}}{\partial x_{i}}\right)^{2} \frac{\partial^{2} x_{s}^{\prime}}{\partial x_{j}^{2}}+\left(\frac{\partial x_{s}^{\prime}}{\partial x_{j}}\right)^{2} \frac{\partial^{2} x_{s}^{\prime}}{\partial x_{i}^{2}} .
$$

So we see that $x_{s}^{\prime}$ is the solution of the corresponding Batemann equation with respect to each pair of arguments $i, j$. 


\section{The simplest case $D=2$}

In this case we have only one pair of variables $x_{1,2}$, and equations (3.4) take the form

$$
x_{1}^{\prime}=\phi(2) x_{2}^{\prime}+\Phi(2), \quad x_{1}^{\prime}=f(1) x_{2}^{\prime}+F(1),
$$

where $\phi(2) \equiv \phi\left(x_{2}\right)$ and so on. Or

$$
x_{2}^{\prime}=-\frac{\Phi(2)-F(1)}{\phi(2)-f(1)}, \quad x_{1}^{\prime}=-\frac{\frac{\Phi(2)}{\phi(2)}-\frac{F(1)}{f(1)}}{\frac{1}{\phi(2)}-\frac{1}{f(1)}} .
$$

Each of the functions $x_{1}^{\prime}, x_{2}^{\prime}$ must satisfy the Batemann equation. Let us write this equation at first for function $x_{2}^{\prime}$. Technically it is more convenient to introduce the function $\theta=$ $\frac{\partial x_{2}^{\prime} / \partial 1}{\partial x_{2}^{\prime} / \partial 2}$ in terms of which the Batemann equation may be rewritten in the form

$$
\frac{\partial \ln \theta}{\partial 1}=\theta \frac{\partial \ln \theta}{\partial 2}
$$

In our case

$$
\theta=-\frac{f_{1}}{\phi_{2}}\left[\frac{\Phi-F-F_{f}(\phi-f)}{\Phi-F-\Phi_{\phi}(\phi-f)}\right],
$$

where $f_{1} \equiv \frac{\partial f}{\partial x_{1}}, \phi_{2} \equiv \frac{\partial \phi}{\partial x_{2}}$ and functions $F, \Phi$ are considered as the functions of arguments $f, \phi$, respectively. The Batemanns equation takes the form

$$
\begin{gathered}
\frac{f_{11}}{f_{1}}+\frac{f_{1} F_{f f}}{\Phi-F-F_{f}(\phi-f)}-\frac{\left(\Phi_{\phi}-F_{f}\right) f_{1}}{\Phi-F-\Phi_{\phi}(\phi-f)}= \\
-\frac{f_{1}}{\phi_{2}}\left[\frac{\Phi-F-F_{f}(\phi-f)}{\Phi-F-\Phi_{\phi}(\phi-f)}\right]\left[-\frac{\phi_{22}}{\phi_{2}}+\frac{\left(\Phi_{\phi}-F_{f}\right) \phi_{2}}{\Phi-F-F_{f}(\phi-f)}-\frac{\Phi_{\phi \phi} \phi_{2}}{\Phi-F-\Phi_{\phi}(\phi-f)}\right] .
\end{gathered}
$$

From (4.2) it follows immediately that $F, \Phi$ are linear functions of their arguments

$$
F=A f+B, \quad \Phi=\alpha \phi+\beta
$$

and

$$
\frac{f_{11}(a f+b)}{f_{1}^{2}}=\frac{\phi_{22}(a \phi+b)}{\phi_{2}^{2}}=D a=\text { const }, \quad a \equiv \alpha-A, \quad b \equiv \beta-B .
$$

First integrals of the last equations have the form

$$
f_{1}=l(a f+b)^{D}, \quad \phi_{2}=m(a \phi+b)^{D},
$$

where $l, m$ - are the constants of integration. So (4.3) and (4.4) have solved the problem of finding $x_{2}^{\prime}$ from (4.1).

The function $x_{1}^{\prime}$ must also be the solution of the Batemann equation. Repeating step by step the above calculations we come to the following equation

$$
\frac{\left(\frac{1}{f}\right)_{11}\left(a+b f^{-1}\right)}{\left(\frac{1}{f}\right)_{1}^{2}}=\frac{\left(\frac{1}{\phi}\right)_{22}\left(a+b \phi^{-1}\right)}{\left(\frac{1}{\phi}\right)_{2}^{2}} .
$$


Keeping in mind the relations (4.4) we conclude that $D=2$. We obtain finally

$$
\frac{1}{a f+b}=k x_{1}+l, \quad \frac{1}{a \phi+b}=m x_{2}+n,
$$

where $k, l, m, n$ are the constants of integration. After substitution of all these results into (4.1) we obtain

$$
x_{2}^{\prime}=-\frac{\alpha k x_{1}-A m x_{2}+\alpha l-n A}{k x_{1}-m x_{2}+l-n}, \quad x_{1}^{\prime}=-\frac{\beta k x_{1}-B m x_{2}+1-B n+\beta l}{k x_{1}-m x_{2}+l-n} .
$$

\section{The general case}

All computations of the previous section are correct with respect to each pair of variables $\left(x_{i}, x_{j}\right)$, and so the general solution of the system (3.5) is the fractional-linear transformation with respect to all the variables of the problem. So we obtain

$$
x_{i}^{\prime}=\frac{(A x)_{i}+B_{i}}{\sum l_{s} x_{s}+l_{D+1}}=\frac{(\tilde{A} y)_{i}}{(\tilde{A} y)_{D+1}}
$$

where $A$ - is an arbitrary $D D$ matrix, $B_{i}$ are the components of a $D$-dimensional column, $l_{k}$ - the components of $D+1$ dimensional row, $\tilde{A}$ is the $(D+1)(D+1)$ matrix, and $y_{s}$ are the components of a $D+1$ dimensional column $\left(y_{1}, y_{2}, \ldots, y_{D}, 1\right)$.

Formula (5.1) may be considered as a realization of transformations of the $S L(D+1, R)$ group (in the case of real coordinates) from one hand, and as a projective transformation of the $D$-dimensional plane from the other.

It is not difficult to check, by direct calculations, that the Universal equation is invariant with respect to transformations (5.1) but in the order to prove that (5.1) is the maximal geometrical symmetry of this equation, the considerations and calculations of the previous sections should be taken into account.

\section{Concluding remarks}

The main result of this paper consists in the proof of the fact that the maximal group of geometrical symmetry of the Universal equation in D-dimensional space coincides with $S L(D+1, R)$ - the group of projective transformations of D-dimensional space. The hidden symmetries of dynamical systems have as their corollary the additional number of conservation laws which in its turn allow to construct a more wide class of exact solutions for the systems of such a type. As it was understood recently [3], the solution of the Universal equation has a direct relation to the definite class of solutions of harmonical and bi-harmonical equations in four dimensions. The discovered maximal group of geometrical symmetry of the Universal equation may be used for these purposes.

The authors thank D.B. Fairlie for the discussion of the results of this paper.

One of the authors (A.N.L.) is indebted to the Soros foudation for the financial support. 


\section{References}

[1] Fairlie D.B., Govaerts J. and Morzov A., Nuclear Phys.B., 1992, V.373, 214-232.

Fairlie D.B. and Govaerts J., Linearization of Universal Field equations, DTP-92/47, NI-92/011, December 1992.

[2] Zwilinger D., Handbook of differential equations, New York, Acad. Press., 1989.

[3] Leznov A.N., Local solutions of harmonical and bi-harmonical equations, Universal Field Equation and self-dual configurations of Yang-Mills fields in four dimensions, Preprint IHEP-70-94 DTP, 1994.

[4] Leznov A.N., Nonlinear Symmetries of Integrable Systems, J. Sov. Lazer. Research, 1992, V.3-4, $278-288$.

Devchand Ch. and Leznov A.N., Backlund transformation for super-symmetric self-dual theories for semisimple Gauge Groups and hierarchy of $A_{1}$ solutions, Preprint IHEP DTP 92-170, 1992; Comm. Math. Phys., March. 1994. 